\title{
Re: Alternatives to colonoscopy for population- wide colorectal cancer screening
}

Hong Kong Med J 2016;22:191

DOI: $10.12809 / \mathrm{hkmj} 164847$

To the Editor-I much appreciate the timely publication of the review paper, "Alternatives to colonoscopy for population-wide colorectal cancer screening," by Leung et al. ${ }^{1}$ We are facing an ageing population and a large proportion of our elderly patients have coronary and other arterial disorders for which they are prescribed aspirin. If stents have been deployed they are prescribed dual antiplatelet therapy. Direct interventional procedures such as colonoscopy might not be the ideal first-line screening procedure due to the risk of bleeding. Computed tomographic (CT) virtual colonoscopy might be an alternative. The CT imaging may not be comparable with the colonoscope, especially for the sub-centimetre polyp, but it has the advantage of picking up extraluminal lesions like epiploic appendagitis. More importantly the CT imaging also serves to screen the whole abdomen beyond the large intestine. The following case report illustrates this issue.

A 78-year-old retired professor complained of ill-defined pain in the chest. She was diagnosed to have coronary artery disease and two drug-eluting stents were deployed. The discomfort persisted and more medications were given. She became constipated and her haemorrhoids started to bleed. At this stage colonoscopy screening for colorectal cancer was considered but was reckoned inadvisable because of her dual antiplatelet therapy. A CT colonoscopy was done and revealed a few polyps and also a discreet tumour in the wall of the fundus of the stomach. Gastroscopy had likewise been vetoed because of the antiplatelet therapy. Nonetheless, the CT image helped to define the tumour outline to the extent that it was unlike the usual gastric carcinoma. Blood test for a panel of tumour markers showed that chromogranin A was strongly positive, thus narrowing the diagnosis to a gastric neuroendocrine tumour.

The standard treatment of a neuroendocrine tumour is surgical excision and so we are regrettably still faced with the problem of dual antiplatelet therapy and the risk of perioperative haemorrhage.

John SM Leung *, FCSHK, FHKAM (Surgery)

St Paul's Hospital

Causeway Bay, Hong Kong

* Corresponding author: leungsiumanjohn@yahoo.com.hk

\section{Reference}

1. Leung WC, Foo DC, Chan TT, et al. Alternatives to colonoscopy for population-wide colorectal cancer screening. Hong Kong Med J 2016;22:70-7. 